PROCEEDINGS OF THE

AMERICAN MATHEMATICAL SOCIETY

Volume 134, Number 4, Pages 1183-1187

S 0002-9939(05)08061-5

Article electronically published on September 20, 2005

\title{
THE MINIMAL SPANNING TREE AND THE UPPER BOX DIMENSION
}

\author{
GADY KOZMA, ZVI LOTKER, AND GIDEON STUPP
}

(Communicated by John R. Stembridge)

\begin{abstract}
We show that the $\alpha$-weight of an MST over $n$ points in a metric space with upper box dimension $d$ has a bound independent of $n$ if $\alpha>d$ and does not have one if $\alpha<d$.
\end{abstract}

\section{INTRODUCTION}

The beginning point of this paper is the following old and well-researched theorem in Euclidean combinatorics

Theorem 1. Let $v_{1}, \ldots, v_{n} \in[0,1]^{d}$ and let $T$ be the minimal spanning tree (MST) over $v_{1}, \ldots, v_{n}$. Then

$$
E_{\alpha}(T):=\sum_{e \in T}\|e\|^{\alpha} \leq C(d)^{\alpha} n^{\max (0,1-\alpha / d)} .
$$

We note that the well-known greedy algorithm for the minimal spanning tree (see e.g. CLR91]) shows that the spanning tree minimizing $E_{1}(T)$ is also the spanning tree minimizing $E_{\alpha}(T)$ for any $\alpha$. This fact is true in any graph, not just in the Euclidean case.

This fact was a part of the general lore of the field for many years. The case $\alpha=1$ was discussed in V51, FT40, F55, M84 with increasingly improved constants. The case $\alpha=d$ (from which the case of general $\alpha$ follows immediately, if one does not care about optimizing the constant) was first proved in the case $d=2$ in GP68 and in the general case is usually attributed to [SS87, even though the result is not proved there explicitly. An almost trivial argument using the existence of a space filling curve with precise Hölder exponent can be found in the survey [S90, page 759], and a different approach is presented in [Y96, L02. We cannot resist citing the beautiful work done on the case of randomly distributed points; see for example A96, ABNW99, AS04. However, we will skip the vast literature on related problems of combinatoric optimization such as the traveling salesperson, minimal matching, greedy matching, Steiner minimal tree and so on.

Examine the proof of Gilbert and Pollak GP68. Their approach is as follows: place a rhombus, or diamond, on any edge of the MST with an opening degree of $60^{\circ}$. It turns out that these rhombi are disjoint, which immediately proves the theorem with a constant of $2 \sqrt{3}$. The proof that they are disjoint uses specific

Received by the editors January 7, 2004 and, in revised form, October 25, 2004.

2000 Mathematics Subject Classification. Primary 68U05, 05 C05.

(C)2005 American Mathematical Society 
facts from the geometry of the plane. However, if one weakens the claim to balls centered on the middle of each edge with radius $\frac{1}{10}$ the length of the edge, the proof becomes simpler and purely metric (some assumption of uniform convexity is necessary). For the convinience of the reader, we add a proof of this fact and hence of Theorem 1 in $\$ 2.1$ below.

Since we have a question phrased in metric terms, and a proof which uses very little from the structure of $\mathbb{R}^{d}$, it seems natural to try to extend the result to more general metric spaces, and in particular to spaces with fractal dimensions. Thus we define the "MST dimension" using

$$
\operatorname{dim}_{\mathrm{MST}}(M):=\inf \left\{d: E_{d}(T) \leq C \quad \forall T \text { a MST on points from } M\right\} .
$$

We will prove that the MST dimension is equivalent to the upper box dimension (also known as the upper Minkowsi dimension, or the entropy dimension), namely

Theorem 2. For every metric space $M$,

$$
\operatorname{dim}_{\mathrm{MST}}(M)=\operatorname{dim}_{\mathrm{Box}}(M) .
$$

See [F90] for alternatives to the Hausdorff dimension and the upper box dimension in particular. We note only that it is well known that $\operatorname{dim}_{\text {Haus }} \leq \operatorname{dim}_{\text {Box }}$ and that they are equal for a wide family of "well behaved" sets.

One notable discrepancy remains between Theorem 1 and Theorem 2 which is the critical case of $\alpha=d$. Can Theorem 2 be strengthened to show boundedness in this case, too? The short answer is no. The long answer is that many factors come into play, most notably the connectivity of the fractal. We plan to analyze specific cases in a separate paper.

\section{Proofs}

In the following, $C$ or $C(d)$ refer to constants, which could be different from place to place.

\subsection{The classic result.}

Lemma 1. Let $w_{1}, w_{2} \in \mathbb{R}^{d}$ satisfy $\left\|w_{1}\right\| \geq 1,\left\|w_{2}\right\| \geq 1$ and $\left\|w_{1}-w_{2}\right\| \leq 1$. Then

$$
\left\|\frac{w_{1}+w_{2}}{2}\right\| \geq \frac{\sqrt{3}}{2}
$$

This is an easy exercise in plane geometry, and we leave it to the reader.

Lemma 2. Let $v_{1}, \ldots, v_{n} \in[0,1]^{d}$ and let $T$ be a spanning tree with minimal energy. For every edge $e \in T$, let $B_{e}$ be a ball of radius $\frac{1}{10}\|e\|$ around the center of e. Then the family of balls $\left\{B_{e}\right\}_{e \in T}$ is pairwise disjoint.

Proof. Assume to the contrary that $B_{e_{1}} \cap B_{e_{2}} \neq \emptyset$. We may assume $\left\|e_{1}\right\| \geq\left\|e_{2}\right\|$. Denote $e_{1}=\left(v_{1}, v_{2}\right)$ and $e_{2}=\left(w_{1}, w_{2}\right)$. Using the triangle inequality twice we get

$$
d\left(v_{1}, \frac{w_{1}+w_{2}}{2}\right) \leq d\left(v_{1}, \frac{v_{1}+v_{2}}{2}\right)+\frac{1}{10}\left\|e_{1}\right\|+\frac{1}{10}\left\|e_{2}\right\|<\frac{\sqrt{3}}{2}\left\|e_{1}\right\| .
$$

We now use Lemma 1 for the vectors $\frac{1}{\left\|e_{1}\right\|}\left(w_{i}-v_{1}\right)$. Since the conclusion of the lemma fails, we know that one of its assumptions fails. Since $\left\|w_{1}-w_{2}\right\|=\left\|e_{2}\right\| \leq$ $\left\|e_{1}\right\|$, the failing assumption must be one of the first two, which means that for some $i \in\{1,2\}, d\left(v_{1}, w_{i}\right)<\left\|e_{1}\right\|$. Similarly, for some $j, d\left(v_{2}, w_{j}\right)<\left\|e_{1}\right\|$. Let $T^{\prime}=T \backslash e_{1}$. 
The removal of one edge splits the tree into two connected components. Assume without loss of generality that $v_{1}$ and $w_{1}$ are in the same component, and then $w_{2}$ would be in the same component. Then $T^{\prime \prime}=T^{\prime} \cup\left(v_{2}, w_{j}\right)$ is a spanning tree with lower energy than $T$. This is a contradiction, and the lemma is proved.

Proof of Theorem 1. If $\alpha=d$, then this follows immediately from Lemma 2, since

$$
\sum_{e \in T}\|e\|^{d} \leq C(d)^{d} \sum_{e \in T} \operatorname{Vol}\left(B_{e}\right)=C^{d} \operatorname{Vol}\left(\bigcup_{e \in T} B_{e}\right) \leq C^{d} \operatorname{Vol}\left([-1,2]^{d}\right)=C^{d} .
$$

This also gives the required result for $\alpha>d$ since in this case

$$
E_{\alpha} \leq \max \|e\|^{\alpha-d} E_{d} \leq C(d)^{\alpha} .
$$

If $\alpha<d$ we can use Hölder's inequality (or $l^{p}-l^{q}$ duality) for $p=d / \alpha$ to get

$$
\begin{aligned}
\sum\|e\|^{\alpha} & =\sum e^{\alpha} \cdot 1 \leq\|\| e\left\|^{\alpha}\right\|_{d / \alpha}\|\overrightarrow{1}\|_{d /(d-\alpha)} \\
& =\left(\sum\|e\|^{d}\right)^{\alpha / d} n^{1-\alpha / d} \leq C(d)^{\alpha} n^{1-\alpha / d}
\end{aligned}
$$

Remark. The dependency of $C$ on $d$ can be made explicit: it is enough to take $C(d)=C \sqrt{d}$, where the right-hand $C$ is absolute. This value appears twice: in (2), where we need $C(d)>C \operatorname{Vol}\left(B^{d}\right)^{-1 / d}$, and where the estimate follows from the well-known formula for the volume of the $d$-dimensional ball. Next it appears in (3), where we need $C(d)>C \operatorname{diam}[0,1]^{d}$ and this is clearly $>C \sqrt{d}$. Thus we see that $C(d)=C \sqrt{d}$ is enough in both cases.

2.2. The upper box dimension. We shall use the following definition of the upper box dimension: let $M$ be a metric space, and let $N(\epsilon)$ be the maximal number of disjoint balls of radius $\epsilon$ in $M$. Then

$$
\operatorname{dim}_{\text {Box }}(M):=\varlimsup_{\epsilon \rightarrow 0} \frac{\log N(\epsilon)}{\log 1 / \epsilon} .
$$

Lemma 3. For every metric space $M$,

$$
\operatorname{dim}_{\text {Box }}(M) \leq \operatorname{dim}_{\mathrm{MST}}(M)
$$

Proof. If $\operatorname{dim}_{\text {Box }}(M)=0$, then there is nothing to prove. Therefore assume $\alpha<$ $\operatorname{dim}_{\text {Box }}(M)$. By the definition of the box dimension, it is possible to find sequences of disjoint balls $B\left(v_{1}, \epsilon\right), B\left(v_{2}, \epsilon\right), \ldots, B\left(v_{N(\epsilon)}, \epsilon\right)$ such that

$$
\varlimsup_{\epsilon \rightarrow 0} \epsilon^{\alpha} N(\epsilon)=\infty .
$$

Let $B\left(v_{i}, \epsilon\right)$ be one such collection. Now examine the MST $T$ of the points $v_{i}$. We get that every edge of $T$ is $\geq \epsilon$ therefore $E_{\alpha}(T) \geq \epsilon^{\alpha} N(\epsilon)$, and since this is unbounded we get $\operatorname{dim}_{\mathrm{MST}}(M) \geq \alpha$. Since this is true for every $\alpha<\operatorname{dim}_{\text {Box }}(M)$ the lemma is proved.

Lemma 4. For every metric space $M$,

$$
\operatorname{dim}_{\mathrm{MST}}(M) \leq \operatorname{dim}_{\mathrm{Box}}(M) .
$$


Proof. We may assume $M$ is bounded, since otherwise both dimensions are $\infty$, and by scaling we may assume $\operatorname{diam} M=1$. Assume $\alpha>\operatorname{dim}_{\text {Box }}(M)$ so that we have

$$
N(\epsilon) \leq C(1 / \epsilon)^{\alpha} \quad \forall \epsilon>0
$$

with $N(\epsilon)$ as above.

Recall the greedy algorithm for obtaining the MST: define $T_{0}=\left\{v_{0}\right\}$ and $T_{i}=$ $T_{i-1} \cup\left\{v_{i}^{\prime}\right\}$, where $v_{i}^{\prime}$ is the vertex not in $T_{i}$ closest to $T_{i}$, and the connection is via the shortest edge connecting $v_{i}^{\prime}$ to $T$. For every edge $e \in T$ satisfying $\|e\|>\epsilon$, define $B(e)$ to be a ball of radius $\frac{1}{3} \epsilon$ centered at the vertex of $e$ which entered last into $T$, or in other words, if $e=\left(v_{i}^{\prime}, v_{j}^{\prime}\right)$ and $i<j$, then $B(e)=B\left(v_{j}^{\prime}, \frac{1}{3} \epsilon\right)$. It follows that these balls are disjoint: assume to the contrary that $B(e) \cap B(f) \neq \emptyset$, and assume w.l.o.g. that $e$ was added to the tree before $f$. Denote $v$ as the center of $B(e)$ and $w$ as the center of $B(f)$ and then $\|v-w\|<\frac{2}{3} \epsilon$. Then, when adding $f$, we have that the partially constructed tree contains $v$, and we add $w$ which contradicts the assumption $\|f\|>\epsilon$.

We now use (44), and we get that there are no more than $C \epsilon^{-\alpha}$ edges of length $>\epsilon$ in $T$. Let $\alpha<\beta$. Then

$$
\begin{aligned}
E_{\beta}(T) & =\sum_{e \in T}\|e\|^{\beta}=\sum_{k=0}^{\infty} \sum_{\substack{e \in T \\
2^{-k-1}<\|e\| \leq 2^{-k}}}\|e\|^{\beta} \\
& \leq \sum_{k=0}^{\infty} C\left(2^{k+1}\right)^{\alpha}\left(2^{-k}\right)^{\beta}=C \sum_{k=0}^{\infty} 2^{k(\alpha-\beta)}=C .
\end{aligned}
$$

This holds for any $\beta>\operatorname{dim}_{\text {Box }}(M)$, as we can always find an $\alpha$ between $\operatorname{dim}_{\text {Box }}(M)$ and $\beta$, and the lemma is proved.

Remarks. (1) The similarity between the proofs of Lemmas 4 and 2 is evident. The only obstacle for us in using Lemma 2 directly is that in a general space $M$ there is no notion of a "point on the middle of an edge". Even if we embed $M$ into $\mathbb{R}^{d}$, the middle of the edge might not belong to $M$, so that we cannot use it for the definition of the upper box dimension.

(2) In effect, we have not used the greedy algorithm at all. What we have shown is that the tree generated by the greedy algorithm has bounded $\beta$-weight. Even if we did not know that this tree is the MST, it would definitely follow for the MST which has the minimal $\beta$-weight.

(3) Theorem 2 may be generalized to spaces that satisfy a weak triangle inequality, namely

$$
d(x, y) \leq C(d(x, z)+d(z, y)) .
$$

The proof carries out without any significant change. We remark that all the classic equivalent definitions for the upper box dimension (using covering or packing numbers) are also equivalent when (5) replaces the triangle inequality.

\section{REFERENCES}

[ABNW99] Michael Aizenman, Almut Burchard, Charles M. Newman and David B. Wilson, Scaling limit for minimal and random spanning trees in two dimensions, Random Structures and Algorithms 15:3-4 (1999), 319-367. http://arxiv.org/abs/math.PR/9809145 MR/1716768(2001c:60151) 
[AS04] David Aldous and J. Michael Steele, The objective method: probabilistic combinatorical optimization and local weak convergence, Probability on discrete structures, 1-72, Encyclopaedia Math. Sci., 110, Springer, Berlin, 2004. http://stat-www.berkeley.edu/users/aldous/me101.ps.Z

[A96] Kenneth S. Alexander, The RSW theorem for continuum percolation and the CLT for Euclidean minimal spanning trees, Ann. Appl. Probability 6 (1996), 466-494. MR.1398054 (97f:60204)

[CLR91] Thomas H. Corman, Charles E. Leiserson and Ronald L. Rivest, Introduction to algorithms, MIT Press, 1991.

[FT40] L. T. Fejes-Tóth, Uber einen geometrische Satz, Mathematische Zeitschrift 46 (1940), 83-85. MR0001587 (1:263g)

[F55] L. Few, The shortest path and the shortest road through $n$ points in a region, Mathematika 2 (1955), 141-144. MR.0078715 (17:1235f)

[F90] K. Falconer, Fractal geometry, mathematical foundations and applications, John Wiley \& Sons, New York, 1990. MR1102677 (92j:28008)

[GP68] E. N. Gilbert and H. O. Pollak, Steiner random minimal trees, SIAM J. Appl. Math. 16 (1968), 1-29. MR0223269 (36:6317)

[L02] Sungchul Lee, Worst case asymptotics of power-weighted Euclidean functionals, Discrete Math. 256:1-2 (2002), 291-300. http://suny.yonsei.ac.kr/ filab/paper/9.ps MR/1927073(2003g:05076)

[M84] S. Moran, On the length of optimal TSP circuits in sets of bounded diameter, J. Combinatorial Theory Ser. B 37 (1984), 113-141. MR0766629 (86i:05087)

[SS87] T. L. Snyder and J. Michael Steele, Worst case growth rates for minimal and greedy matchings with power weighted edges, Technical report, Princeton University, Program in Operations Research and Statistics (1987).

[S90] J. Michael Steele, Probabilistic and worst case analyses of classical problems of combinatorial optimization in Euclidean space, Mathematics of Operations Research 15:4 (1990), 749-770. MR.1080476 (92e:68080)

[V51] S. Verblunsky, On the shortest path through a number of points, Proc. Amer. Math. Soc. 2 (1951), 904-913. MR0045403(13:577c)

[Y96] Joseph E. Yukich, Worst case growth rates for some classical optimization problems, Combinatorica 16 (1996), 575-586. MR1433644 (97i:90061)

Institute for Advanced Study, 1 Einstein Drive, Princeton, New Jersey 08540

E-mail address: gady@ias.edu

Office Centrum voor Wiskunde en Informatica, Kruislaan, 413, NL-1098 SJ AmsterDAM, The Netherlands

E-mail address: zvilo@eng.tau.ac.il

mit Computer Science and Artificial Intelligence laboratory, The Stata Center, 32 Vassar Street, Cambridge, Massachusetts 02139

E-mail address: gstupp@theory.csail.mit.edu 